\title{
ANALYSIS OF EXPRESSION OF GENES RESPONSIBLE FOR REGULATION OF CELLULAR PROLIFERATION AND MIGRATION - MICROARRAY APPROACH BASED ON PORCINE OOCYTE MODEL
}

Agata Chamier-Gliszczyńska ${ }^{1}$, Sandra Kałużna ${ }^{1}$, Katarzyna Stefańska ${ }^{1}$, Piotr Celichowski ${ }^{1}$, Paweł Antosik $^{2}$, Dorota Bukowska ${ }^{2}$, Małgorzata Bruska ${ }^{3}$, Jana Zakova ${ }^{4}$, Marie Machatkova ${ }^{5}$, Michal Jeseta $^{4}$, Michał Nowicki ${ }^{1}$

\begin{abstract}
The formation of mammalian oocytes begins in the ovary during fetal development. The proper development of oocytes requires close communication with surrounding somatic cells, the substances they emit allow proper maturation of oocytes. Somatic cumulus (CC) cells and oocytes form cumulus-oocyte (COC) complexes.

In this study, the Affymetrix microarray analysis was used to investigate changes in gene expression occurring in oocytes before and after in vitro maturation (IVM). The aim of the study was to examine oocyte genes involved in two ontological groups, "regulation of cell migration" and "regulation of cell proliferation" discovered by the microarray method.

We found a reduced expression of all 28 genes tested in the ontological groups: ID2, VEGFA, BTG2, CCND2, EDNRA, TGFBR3, GJA, LAMA2, RTN4, CDK6, IHH, MAGED1, INSR, CD9, PTGES, TXNIP, ITGB1, SMAD4, MAP3K1, NOTCH2 , IGFBP7, KLF10, KIT, TPM1, PLD1, BTG3, CD47 and MITF. We chose the most regulated genes down the IVM culture, and pointed out those belonging to two ontological groups.

Increased expression of the described genes before IVM maturation may indicate the important role of these genes in the process of ovum maturation. After the maturation process, the proteins produced by them did not play such an important role. In summary, the study provides us with many genes that can serve as molecular markers of oocyte processes associated with in vitro maturation. This knowledge can be used for detailed studies on the regulation of oocyte maturation processes.
\end{abstract}

Running title: Genes regulating cellular migration and proliferation in porcine oocytes

Keywords: pig, oocyte, cell, proliferation, migration

\footnotetext{
${ }^{1}$ Department of Histology and Embryology, Poznan University of Medical Sciences, Poznan, Poland

${ }^{2}$ Veterinary Centre, Nicolaus Copernicus University in Torun, Torun, Poland

${ }^{3}$ Department of Anatomy, Poznan University of Medical Sciences, Poznan, Poland

${ }^{4}$ Department of Obstetrics and Gynecology, University Hospital and Masaryk

University, Obilnitrh 11, 60200 Brno, Czech Republic

${ }^{5}$ Veterinary Research Institute, Brno, Czech Republic

* Correspondence: mnowicki@ump.edu.pl

Full list of author information is available at the end of article
} 


\section{Introduction}

The mammalian oocytes formation begins in the ovary during the fetal development. In the perinatal period the primary oocyte is surrounded by the epithelial cells which form a primordial follicle [1]. After oocytes start maturation, their nuclei-germinal vesicles (GV) break down and chromosomes condense [2]. Each oocyte in the ovary is arrested by inhibitory signals in the prophase I during the first meiotic division [3]. This arrest occurs after chromosome pairing and crossing-over process between parental chromosomes [4] The factors that maintain the meiotic arrest of the oocytes originate from the surrounding follicle $[1,5]$. The oocytes arrested at the GV stage, require hormonal stimulation to resume the meiosis. The gonadotrophin signal induces the exit from the prophase I arrest and reinitiate the maturation process [4,5]. Naturally, fully grown mammalian oocytes are arrested at two points of maturation. The second arrest takes place in the metaphase II stage during the puberty. The maturation is completed when oocytes reach MII stage and are ready for fertilization [6]. The fertilization process stops the second arrest and terminates meiosis. The duration of oocyte full maturation is dependent on the species, in humans it can last decades [4]. In in vitro conditions, nuclear maturation is hormone-independent and is initiated by removing the oocytes from their follicular environment [5].

The proper oocyte development requires close communication with surrounding somatic cells. These cells are affected by wide range of regulatory molecules, proteins and hormones leading to proper oocyte maturation. The somatic cumulus cells (CCs) and oocytes form cumulus-oocyte complexes (COCs). They strongly communicate with each other in both directions due to molecular connections, like gap-junctions, between them [7]. Small molecules like amino acids or nucleotides are transported through the junctions and are crucial for proper oocyte development. An oocyte produces many growth factors which contribute to proliferation and differentiation of cumulus cells and prevent them from differentiating into a different type of cells $[8,9]$.One of the most essential transferred macromolecules are nucleic acids, especially RNA [10]. Interruption of the junctions results in inhibition of meiosis resumption and thus stopping the oocyte maturation [11]. It has been discovered that only oocytes, tightly surrounded by cumulus cells, are able to become fully mature, thus the COCs formation is a crucial step at the time of early oogenesis [12].

In this study, the microarray analysis was used to investigate the gene expression changes occurring in the oocytes before and after in vitro maturation (IVM). The oocytes transcriptome analysis could help to understand the events happening during oocytes development and improve knowledge about relationships between oocyte and cu- mulus cells. The aim of the study was to examine the oocyte genes involved in two ontology groups, "regulation of cell migration" and "regulation of cell proliferation" discovered using microarray method. Our results could provide an information about the interactions within COCs and propose new markers for cellular migration and proliferation.

\section{Material and Methods \\ Animals}

A total of 45 pubertal crossbred Landrace gilts bred on a local, commercial farm were used in this study. They had a mean age of 155 days (range 140170 days) and weight of $100 \mathrm{~kg}(95-120 \mathrm{~kg})$. All of the animals were housed under identical conditions and fed the same forage (depending on age and reproductive status).

\section{Collection of porcine ovaries and COCs}

Ovaries and reproductive tracts were recovered at slaughter and transported to the laboratory at $38^{\circ} \mathrm{C}$ in $0.9 \% \mathrm{NaCl}$ within $40 \mathrm{~min}$. To provide optimal conditions for subsequent oocyte maturation and fertilization in vitro, the ovaries of each animal were placed in $5 \%$ fetal bovine serum solution (Sigma-Aldrich Co., St. Louis, MO) in phosphate-buffered saline (PBS). Then, single large follicles ( $>5$ $\mathrm{mm}$ ) were opened by puncturing with a $5 \mathrm{~mL} \mathrm{sy-}$ ringe and $20-G$ needle in a sterile petri dish, and COCs were recovered. The COCs were washed three times in modified PBS supplemented with $36 \mathrm{mg} /$ $\mathrm{mL}$ pyruvate, $50 \mathrm{mg} / \mathrm{mL}$ gentamycin, and $0.5 \mathrm{mg} /$ $\mathrm{mL}$ bovine serum albumin (BSA; Sigma-Aldrich). COCs were selected under an inverted microscope - Zeiss, Axiovert 35 (Lübeck, Germany), counted and morphologically evaluated. Only COCs of grade I with homogeneous ooplasm and uniform, compact CCs were considered for the following steps of the experiment, resulting in 300 grade I oocytes $(3 \mathrm{x}$ $\mathrm{n}=50$ before IVM, $3 \times \mathrm{n}=50$ after IVM).

\section{Assessment of oocyte developmental competence by brilliant cresyl blue test}

To perform the brilliant cresyl blue (BCB) staining test, oocytes were washed twice in modified Dulbecco PBS (DPBS; Sigma-Aldrich) supplemented with $50 \mathrm{IU} / \mathrm{mL}$ penicillin, $50 \mathrm{mg} / \mathrm{mL}$ streptomycin (Sigma-Aldrich), 0.4\% BSA (w/v), $0.34 \mathrm{mM}$ pyruvate, and $5.5 \mathrm{mM}$ glucose Dulbecco's phosphate buffered saline modified (DPBSm). Thereafter, they were treated with $13 \mathrm{mM}$ BCB (Sigma-Aldrich) diluted in DPBSm at $38.5^{\circ} \mathrm{C}$ and $5 \% \mathrm{CO} 2$ for $90 \mathrm{~min}$. After treatment, the oocytes were transferred to DPBSm and washed twice. During the washing procedure, the oocytes were examined under an inverted microscope and classified as either stained blue $(\mathrm{BCB}+)$ or colourless (BCB-). The collected pool comprised $55 \%$ of $\mathrm{BCB}+$ and $45 \%$ of BCB- oocytes. Immature oocytes have compact CC layers that re- 
quire removal for further oocyte evaluation. Hence, the BCB+ COCs were first incubated with bovine testicular hyaluronidase (Sigma-Aldrich) for $2 \mathrm{~min}$ at $38^{\circ} \mathrm{C}$ to separate CCs and GCs. Cells were then removed by vortexing the $\mathrm{BCB}+$ oocytes in $1 \%$ sodium citrate buffer followed by mechanical displacement using a small-diameter glass micropipette. Only the $\mathrm{GC}$-free $\mathrm{BCB}+$ oocytes were used for subsequent IVM and microarray analysis.

\section{IVM of porcine COCs}

After the first BCB test, the COCs with stained blue cytoplasm (BCB+) were cultured in $\operatorname{Nunclon}^{\mathrm{TM}} \Delta$ four-well dishes in $500 \mathrm{~mL}$ of standard porcine IVM culture medium TCM-199 (tissue culture medium) with Earle's salts and l-glutamine (Gibco BRL Life Technologies, Grand Island, NY) supplemented with $2.2 \mathrm{mg} / \mathrm{mL}$ sodium bicarbonate (NacalaiTesque, Inc., Kyoto, Japan), $0.1 \mathrm{mg} / \mathrm{mL}$ sodium pyruvate (Sigma-Aldrich), $10 \mathrm{mg} / \mathrm{mL}$ BSA (Sigma-Aldrich), $0.1 \mathrm{mg} / \mathrm{mL}$ cysteine (Sigma-Aldrich), 10\% filtered porcine follicular fluid ( $\mathrm{v} / \mathrm{v})$, and gonadotropin supplements at final concentrations of $2.5 \mathrm{IU} / \mathrm{mL}$ human chorionic gonadotropin (Ayerst Laboratories, Inc., Philadelphia, PA) and $2.5 \mathrm{IU} / \mathrm{mL}$ equine chorionic gonadotropin (Intervet, Whitby, Canada). Wells were covered with a mineral oil overlay and cultured for $44 \mathrm{~h}$ at $38^{\circ} \mathrm{C}$ under $5 \%$ CO2. After cultivation, the BCB staining test was performed again, and $\mathrm{BCB}+$ oocytes were used for further experiments.

\section{RNA extraction from porcine oocytes}

Oocytes investigated before and after IVM were pooled into three independent samples for each experimental group. Total RNA was extracted from samples using TRI Reagent (Sigma, St. Louis, MO) and RNeasy MinElutecleanup kit (Qiagen, Hilden, Germany). The amount of total mRNA was determined from the optical density (OD) at $260 \mathrm{~nm}$, and the RNA purity was estimated using the $260 / 280 \mathrm{~nm}$ absorption ratio (higher than 1.8) (NanoDrop spectrophotometer; Thermo Scientific, ALAB, Poland). The RNA integrity and quality were checked on a Bioanalyzer 2100 (Agilent Technologies, Inc., Santa Clara, CA). The resulting RNA integrity numbers were between 8.5 and 10 with an average of 9.2 (Agilent Technologies, Inc.). The RNA in each sample was diluted to a concentration of $100 \mathrm{ng} / \mathrm{mL}$ with an OD260/OD280 ratio of 1.8/2.0. From each RNA sample, $500 \mathrm{ng}$ of RNA was taken for microarray expression assays.

\section{Microarray expression analysis and statistics}

Total RNA (100 ng) from each pooled sample was subjected to two rounds of sense cDNA amplification (Ambion $₫$ WT Expression Kit). The obtained cDNA was used for biotin labeling and fragmentation using Affymetrix GeneChip ${ }^{\circ}$ WT Terminal Labeling and Hybridization (Affymetrix, Santa Clara, CA, USA). Biotin-labeled fragments of cDNA $(5.5 \mu \mathrm{g})$ were hybridized to the Affymetrix $₫$ Porcine Gene 1.1 ST Array Strip $\left(48^{\circ} \mathrm{C} / 20 \mathrm{~h}\right)$. Microarrays were then washed and stained, according to the technical protocol, using the Affymetrix GeneAtlas Fluidics Station. The array strips were scanned employing the Imaging Station of the GeneAtlas System. Preliminary analysis of the scanned chips was performed using Affymetrix GeneAtlas ${ }^{\mathrm{TM}}$ Operating Software. The quality of gene expression data was confirmed according to the quality control criteria provided by the software. The obtained CEL files were imported into downstream data analysis software.

All of the presented analyses and graphs were compiled using Bioconductor and R programming languages. Each CEL file was merged with a description file. To correct background, normalize, and summarize results, we used the Robust Multiarray Averaging (RMA) algorithm. To determine the statistical significance of the analyzed genes, moderated t-statistics from the empirical Bayes method were performed. The obtained p-value was corrected for multiple comparisons using Benjamini and Hochberg's false discovery rate. Selection of significantly altered genes was based on a p-value beneath 0.05 and expression higher than two-fold.

Differentially expressed genes were subjected selection by examination of genes involved in oxygen metabolism. The differentially expressed gene list (separated for up- and down-regulated genes) was uploaded to the DAVID software (Database for Annotation, Visualization and Integrated Discovery) [13], where genes belonging to the terms of all four Gene Ontologies (GOs) of interest were extracted. Expression data of these genes was also subjected to a hierarchical clusterization procedure, with their expression values presented as a heat map.

Subsequently, we analyzed the relation between the genes belonging to the chosen GO terms using theGOplot package [14]. The GoPlot package had calculated the z-score: the number of up-regulated genes minus the number of down-regulated genes divided by the square root of the count. This information allowed to estimate the change course of each gene-ontology term.

Interactions between differentially expressed genes/proteins belonging to the studied gene ontology groups were investigated by the STRING10 software (Search Tool for the Retrieval of Interacting Genes) [15]. The list of gene names was used as a query for interaction prediction. The search criteria were based on co-occurrences of genes/proteins in scientific texts (text mining), co-expression, and experimentally observed interactions. The results of such analyses generated a gene/protein interaction network where the intensity of the edges reflected the strength of the interaction score.

Finally, the functional interactions between genes that belongs to the chosen GO BP terms were investigated by the REACTOME FIViz application to the 
Cytoscape 3.6.0 software. The ReactomeFIViz app is designed to find pathways and network patterns related to cancer and other types of diseases. This app accesses the pathways stored in the Reactome database, allowing to perform pathway enrichment analysis for a set of genes, visualize hit pathways using manually laid-out pathway diagrams directly in Cytoscape, and investigate functional relationships among genes in hit pathways. The app can also access the Reactome Functional Interaction (FI) network, a highly reliable, manually curated pathway-based protein functional interaction network covering over $60 \%$ of human proteins.

\section{Ethical approval}

The research related to animal use has been complied with all the relevant national regulations and instructional policies for the care and use of animals. Bioethical Committee approval no. 83/2012/DNT.

\section{Results}

Whole transcriptome profiling withAffymetrix microarrays allowsus to analyze the gene expression changes between 7, 15 and 30 days of porcine oviductal epithelial cell culture. Using Affymetrix $\AA$ Porcine Gene 1.1 ST Array Strip, we have examined the expression of 12258 transcripts. Genes with fold change higher than abs (2) and with corrected p-value lower than 0.05 were considered as differentially expressed. This set of genes consists of 419 different transcripts.

DAVID (Database for Annotation, Visualization and Integrated Discovery) software was used for extraction of gene ontology biological process terms
(GO BP) that contain differently expressed transcripts. Up and down regulated gene sets were subjected to the DAVID search separately and only gene sets with adj. p-value lower than 0.05 were selected. The DAVID software analysis showed that the differently expressed genes belonged to 657 Gene ontology terms. In this paper, we focused on 28 genes that belong to "regulation of cell migration" and "regulation of cell proliferation"GO BP terms. These sets of genes were subjected to hierarchical clusterization procedure and presented as heatmaps (Fig. 1). The gene symbols, fold changes in expression, Entrez gene IDs and corrected p-values of these genes were shown in table 1.

The enrichment of each GO BP term was calculated as a z-score and shown on the circle diagram (Fig. 2).

The chosen GO BP terms contain 166 differently expressed genes. Therefore, we calculated the mean fold change ratio value of each gene between 7,15 and 30 days of culture. Based on that criteria, we chose the 10 most downregulated and 10 most upregulated genes for further analysis.

In Gene Ontology database, genes that form one particular GO can also belong to other GO term categories. For this reason, we explored the gene intersections between the selected GO BP terms. The relation between those GO BP terms was presented as circle plot (Fig. 3) as well as heatmap (Fig. 4).

STRING interaction network was generated among the differentially expressed genes belonging to each of the selected GO BP terms. Using such prediction method provided us with a molecular interaction network formed between protein products regulation of cell migration

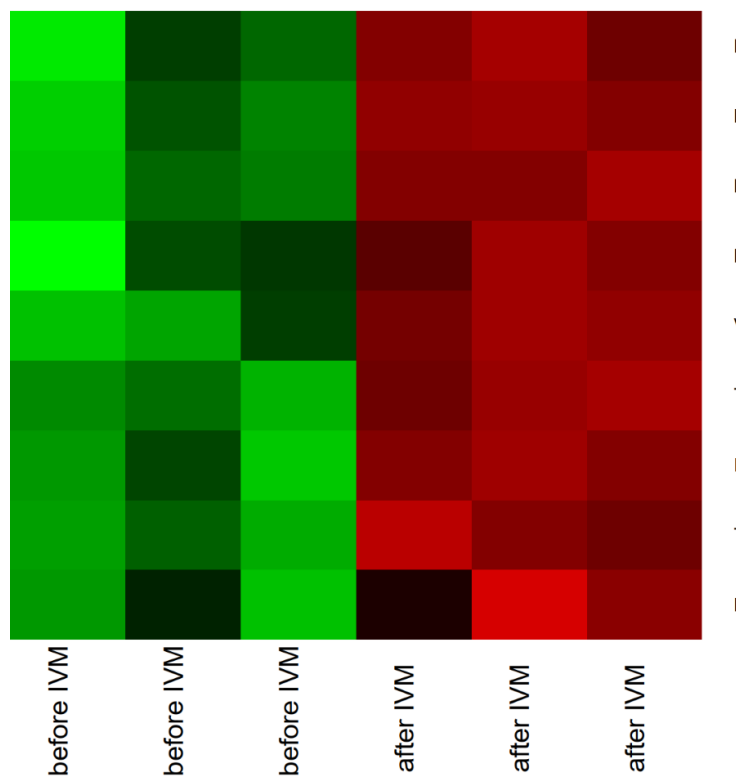

regulation of cell proliferation

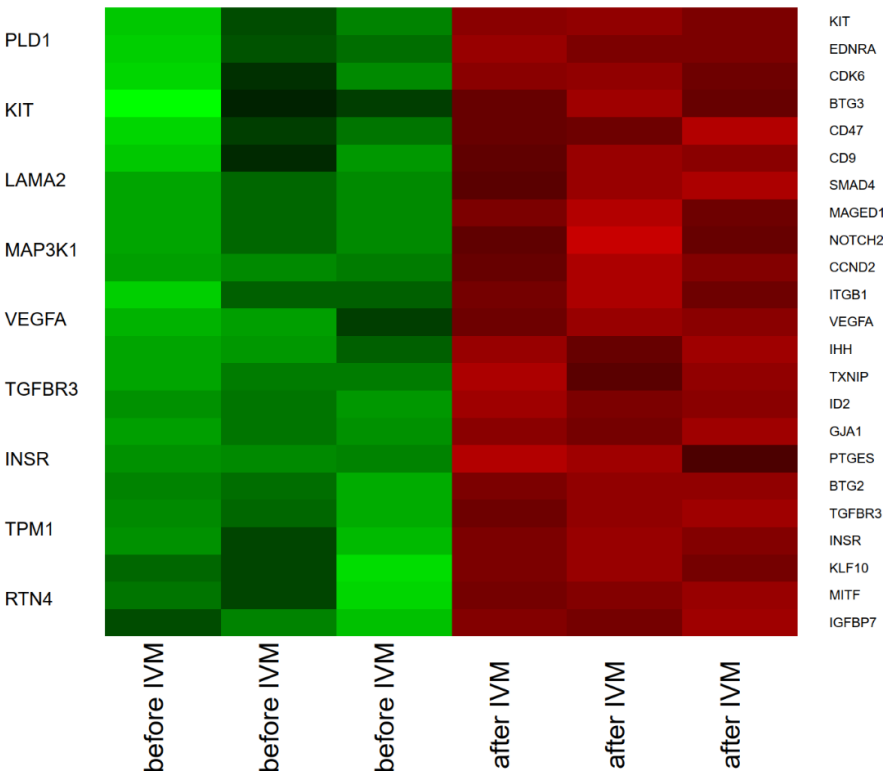

FIGURE 1 Heat map representations of differentially expressed genes belonging to the "regulation of cell migration" and "regulation of cell proliferation" GO BP terms. Arbitrary signal intensity acquired from microarray analysis is represented by colors (green, higher; red, lower expression). Log2 signal intensity values for any single gene were resized to Row Z-Score scale (from -2 , the lowest expression to +2 , the highest expression for single gene) 
TABLE 1 Gene symbols, fold changes in expression, corrected p values and LogFC of studied genes

\begin{tabular}{|c|c|c|c|}
\hline GENE SYMBOL & FOLD CHANGE & ADJUSTEDP, VALUE & LOGFC \\
\hline ID2 & 0,063 & $4,74^{*} 10^{\wedge} 5$ & $-1,201$ \\
\hline VEGFA & 0,070 & $1,91 * 10^{\wedge} 3$ & $-1,157$ \\
\hline BTG2 & 0,074 & $9,55^{*} 10^{\wedge} 5$ & $-1,129$ \\
\hline CCND2 & 0,122 & $1,79 * 10^{\wedge} 4$ & $-0,914$ \\
\hline EDNRA & 0,167 & $1,85^{*} 10^{\wedge} 3$ & $-0,777$ \\
\hline TGFBR3 & 0,197 & $4,06^{*} 10^{\wedge} 4$ & $-0,707$ \\
\hline GJA1 & 0,207 & $1,08^{*} 10^{\wedge} 4$ & $-0,684$ \\
\hline LAMA2 & 0,220 & $7,95 * 10^{\wedge} 4$ & $-0,658$ \\
\hline RTN4 & 0,231 & $2,75^{*} 10^{\wedge} 2$ & $-0,636$ \\
\hline CDK6 & 0,248 & $6,04 * 10^{\wedge} 3$ & $-0,606$ \\
\hline IHH & 0,305 & $5,51 * 10^{\wedge} 4$ & $-0,516$ \\
\hline MAGED1 & 0,306 & $7,06 * 10^{\wedge} 4$ & $-0,515$ \\
\hline INSR & 0,316 & $1,91 * 10^{\wedge} 3$ & $-0,500$ \\
\hline CD9 & 0,329 & $6,33^{*} 10^{\wedge} 3$ & $-0,482$ \\
\hline PTGES & 0,337 & $1,21 * 10^{\wedge} 3$ & $-0,472$ \\
\hline TXNIP & 0,356 & $7,81 * 10^{\wedge} 4$ & $-0,449$ \\
\hline ITGB1 & 0,366 & $3,71 * 10^{\wedge} 3$ & $-0,436$ \\
\hline SMAD4 & 0,368 & $1,24 * 10^{\wedge} 3$ & $-0,434$ \\
\hline MAPЗK1 & 0,369 & $2,47 * 10^{\wedge} 2$ & $-0,433$ \\
\hline NOTCH2 & 0,385 & $2,52^{*} 10^{\wedge} 3$ & $-0,415$ \\
\hline IGFBP7 & 0,404 & $2,50^{*} 10^{\wedge} 3$ & $-0,394$ \\
\hline KLF10 & 0,405 & $6,85^{*} 10^{\wedge} 3$ & $-0,392$ \\
\hline KIT & 0,430 & $2,56 * 10^{\wedge} 3$ & $-0,366$ \\
\hline TPM1 & 0,434 & $1,63^{*} 10^{\wedge} 3$ & $-0,363$ \\
\hline PLD1 & 0,468 & $1,10^{*} 10^{\wedge} 2$ & $-0,329$ \\
\hline BTG3 & 0,486 & $4,03^{*} 10^{\wedge} 2$ & $-0,314$ \\
\hline$C D 47$ & 0,487 & $9,29 * 10^{\wedge} 3$ & $-0,313$ \\
\hline MITF & 0,492 & $6,33^{*} 10^{\wedge} 3$ & $-0,308$ \\
\hline
\end{tabular}
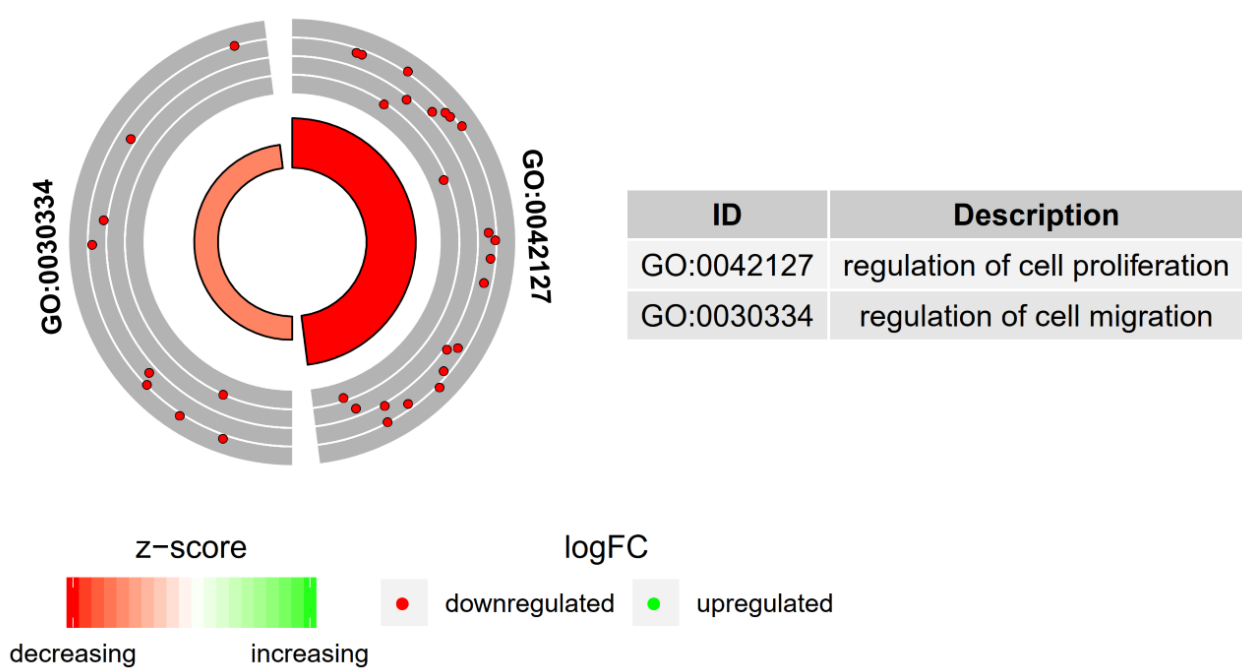

FIGURE 2 The circle plot showing the differently expressed genes and z-scores of "regulation of cell migration" and "regulation of cell proliferation" GO BP terms. The outer circle shows a scatter plot for each term of the fold change of the assigned genes. Greencircles display up- regulation and red ones down- regulation. The inner circle shows the z-score of each GO BP term. The width of each bar corresponds to the number of genes within GO BP term and the color corresponds to the z-score 


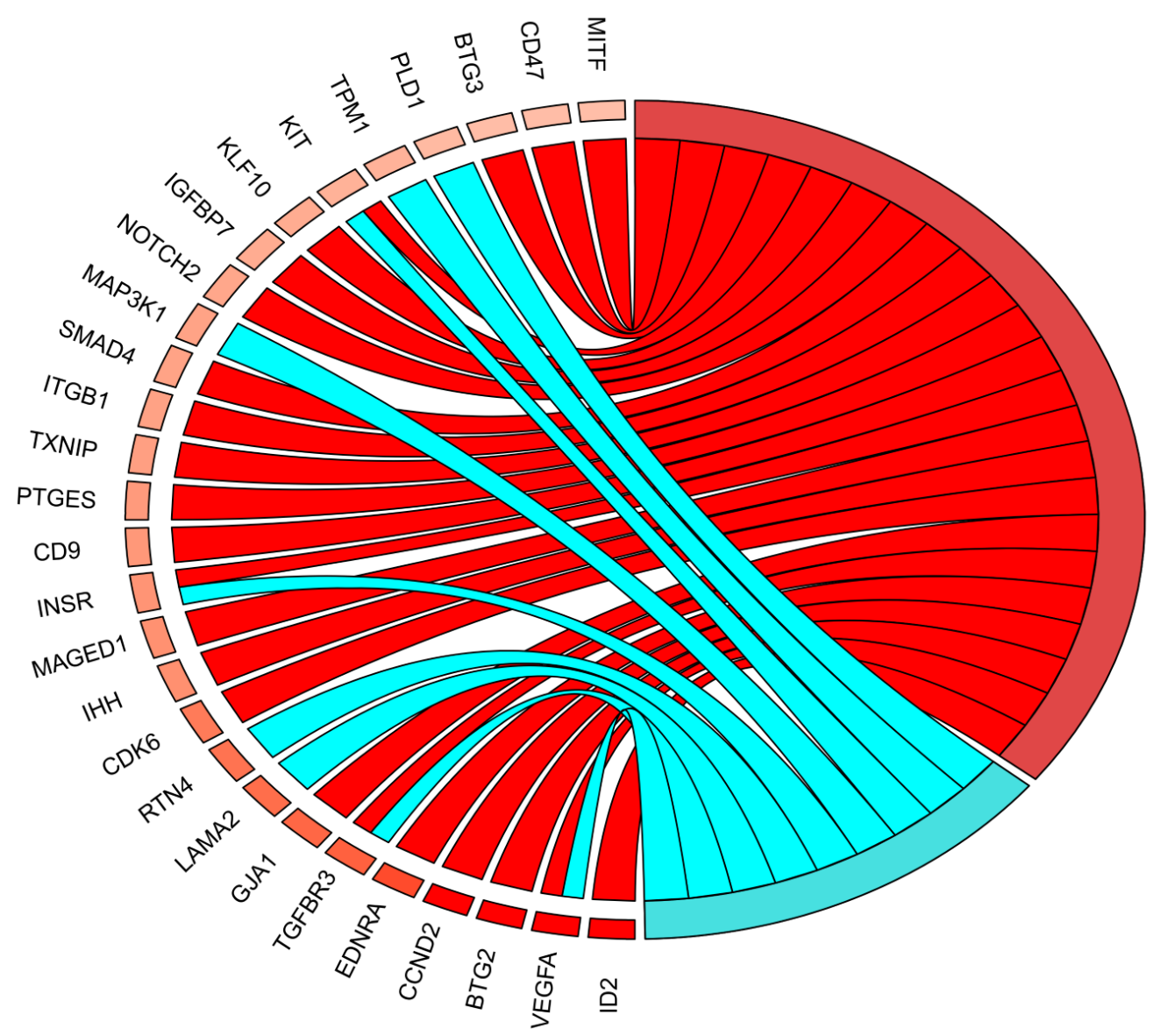

GO Terms $\square$ regulation of cell proliferation $\square$ regulation of cell migration

FIGURE 3 The representation of the mutual relationship between differently expressed genes that belongs to the "regulation of cell migration" and "regulation of cell proliferation" GO BP terms. The ribbons indicate which gene belongs to which categories. The middle circle represents logarithm from fold change (LogFC). The genes were sorted by logFC from most to least changed gene. The color of the each LogFC bar corresponds with LogFC value

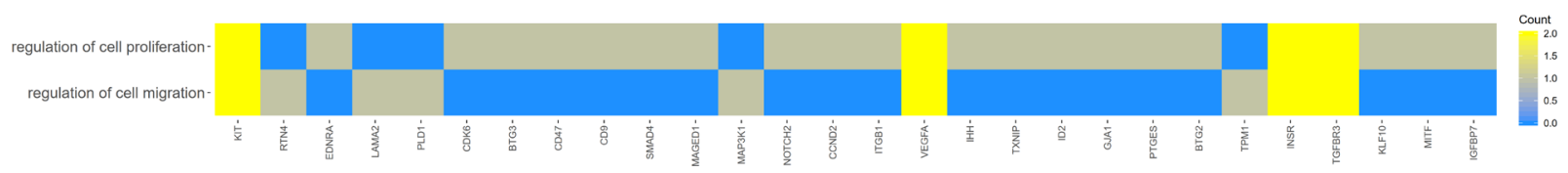

FIGURE 4 Heatmap showing the gene occurrence between differently expressed genes that belongs to the "regulation of cell migration" and "regulation of cell proliferation" GO BP terms. The yellow color is associated with gene occurrence in the GO Term. The intensity of the color is corresponding to amount of GO BP terms that each gene belongs to

of the studied genes (Fig. 5). Finally, we investigated the functional interactions between the chosen genes with REACTOME FIViz app to the Cytoscape 3.6.0 software. The results were shown in (Fig. 6).

\section{Discussion}

In this study, we selected differentially expressed genes from oocytes, using a microarray approach. These genes were classified into 2 ontology groups: "regulation of cell proliferation" and "regulation of cell migration". We investigated the expression of these genes before in vitro maturation and after in vitro maturation, in order to identify new molecular markers associated with the ability of female gametes maturation. All genes constantly changed their expression. All selected genes were first up-regulated and after IVM their expression started decreasing. From 28 differentially expressed genes we chose those which were the most down-regulated after IVM culture and those which interact the most with each other and have the biggest impact on oocyte maturation. We also focused attention on genes 


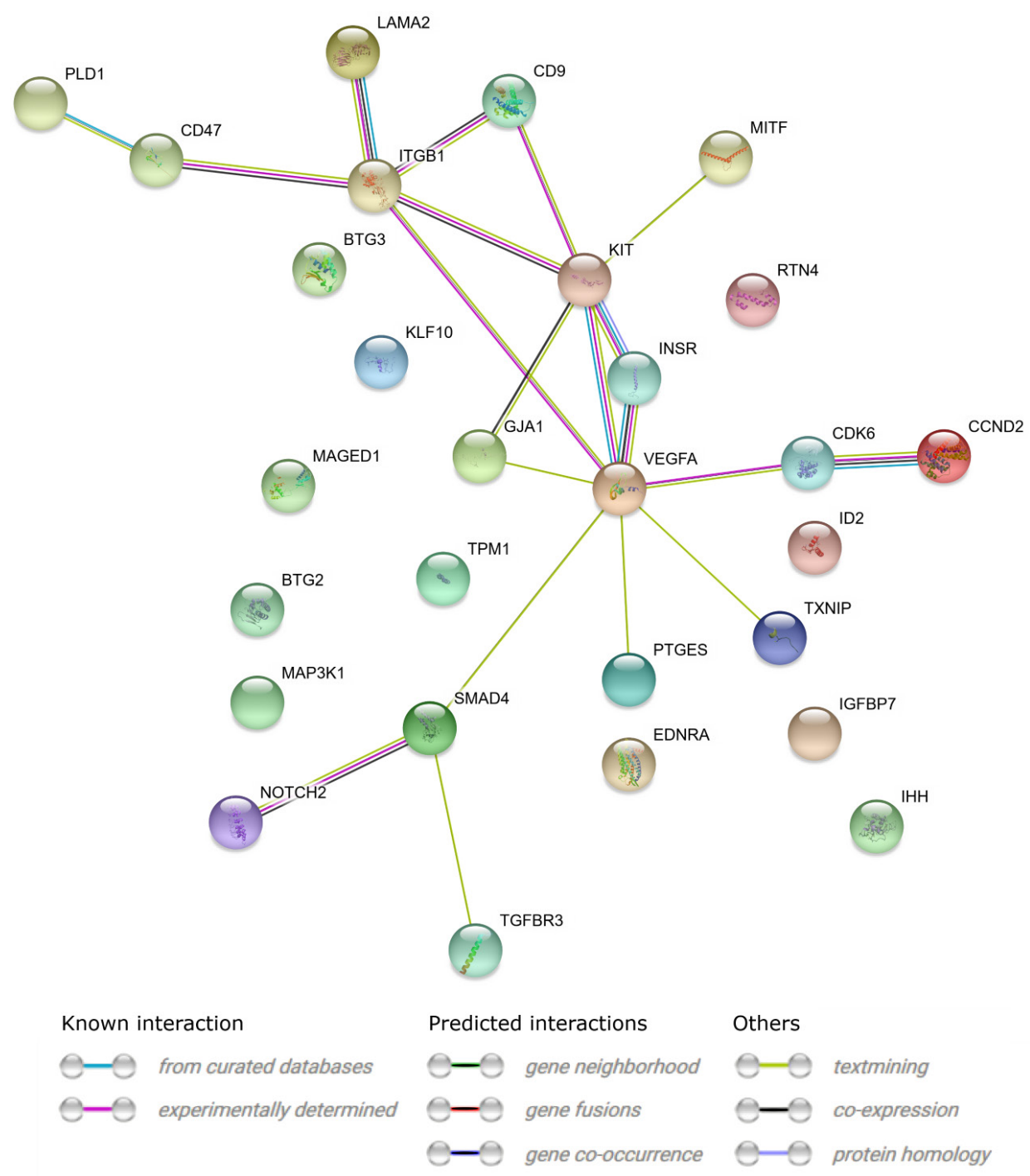

FIGURE 5 STRING-generated interaction network between genes that belongs to the "regulation of cell migration" and "regulation of cell proliferation" GO BP terms. The intensity of the edges reflects the strength of interaction score
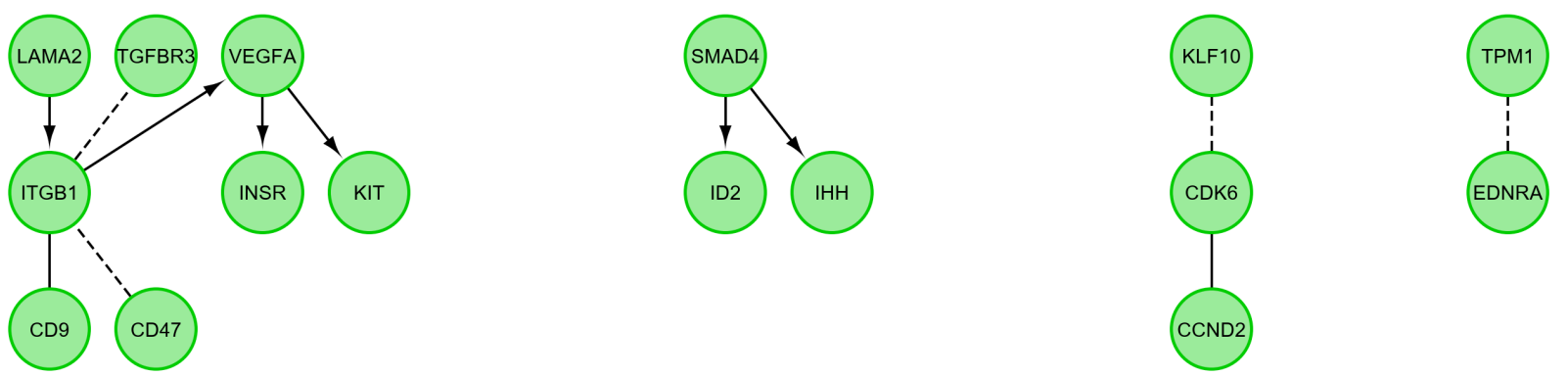

FIGURE 6 Functional interaction (FI) between differently expressed genes that belongs to the "regulation of cell migration" and "regulation of cell proliferation" GO BP terms. In following figure "->" stands for activating/catalyzing, "-|" for inhibition, "-" for FIs extracted from complexes or inputs, and "---" for predicted FIs

belonging to two ontological groups: regulation of cell proliferation and regulation of cell migration.

The most downregulated gene is ID2 (inhibitor of DNA binding 2), it represents only one ontology group, the "regulation of cell proliferation". The protein encoded by ID2 belongs to inhibitor of
DNA-binding (ID) family which members are transcriptional regulators, containing helix-loop-helix (HLH) domain, however lack a basic domain which is crucial for DNA binding. ID2 promotes cell proliferation, which implies its role in negative cell differentiation control $[16,17]$. ID2 protein is known 
of being overexpressed in the cancer cells, it has an important role in development of cancer cells. The studies indicate its participation in pancreatic tumor mass growth or resistance to therapies of the most aggressive brain cancer - glioblastoma [18, 19]. A downregulation of this gene in the oocyte may be related with its role in completion of oocyte maturation and preparing to further fertilization.

The second most downregulated gene is VEGFA (vascular endothelial growth factor A) which belongs to both "regulation of cell proliferation" and " regulation of cell migration" ontology groups. This gene is assigned also to other ontology groups associated with oocyte maturation, like "cell maturation" or "ovarian follicle development", which are not analyzed in this study. VEGFA has a crucial role in angiogenesis and vasculogenesis. It promotes angiogenesis in the ovulatory follicle [20]. The studies on bovine oocytes after in vitro maturation showed that supplementation with VEGF had a positive impact on cytoplasm maturation and oocyte developmental capacity [21]. We discovered that VEGFA interacts with other genes including INSR and KIT, which also belong to both ontology groups (Fig. 6). which is confirmed by the research of our team [22,23].

Another interesting gene is the insulin receptor INSR. Insulin plays an important role in the ovary because it is an important mediator for the development of follicles, steroidogenesis, oocyte maturation as well as embryo development. INSR was first identified in the granular cells of antral human follicles and is the insulin receptor mediating the action of insulin on oocytes. In 2008, INSR was shown to be distributed in all ovarian tissues, including granular and rusty cells, and carcass tissue in cattle. Therefore, it is very often a component of culture media for cells and tissues. Studies conducted by Chaves et al. showed that supplementation of medium with insulin and FSH caused an increase in INSR mRNA levels. Insulin has an effect through its own receptor to cause the response of granulocyte cells to gonadotrophins [22,24]. In our research, the INSR gene ranked 13th in terms of the decrease in expression after IVM. Our research indicates that this gene is important for both ontological groups and has a significant role in the development of oocytes.

The next gene present in both ontological groups is the KIT gene, which is a molecular marker characteristic for oocytes. KIT is proto-oncogene receptor tyrosine kinase [23] and itis an important gene for the survival of germ cells and the development of follicles on their several stages. Many publications report that KIT signalling is important for promoting cell survival, proliferation and differentiation. It has also been shown that the KIT pathway is important for many ovarian functions, including survival and migration of germ cells. In the case of ovarian follicles, it is responsible for the transition from preantral to antral follicle [25]. The Cadoret et al. study showed a decreased KIT expression in in vitro culture of ovarian follicle cells, which was explained by the reduced oocyte growth rate in vitro relative to in vivo [26]. In the case of our studies, we showed a decrease in the expression of the KIT gene after IVM which confirms its role of the proliferation and differentiation of oocytes.

The third most downregulated gene is BTG2 (B-Cell Translocation Gene 2) which is represented only in one of the ontological group:" regulation of cell proliferation". BTG2 is characterized by antiproliferative properties and is part of the gene family that are responsible for development, differentiation and survival, as well as apoptosis, cell death [23]. In the studies of Chermuła et al of the BTG2 gene, a significant decrease in gene expression in the regulation of cell proliferation after IVM was also described. In scientific research, it was noticed that the expression of the BTG2 gene in many human tumors is reduced which is associated with poor cell differentiation. This gene can also cause cell apoptosis. Therefore, the suppressed expression of this gene in the ooze can indicate that oocytes don't differentiate during puberty and do not show the need for apoptosis [27]. Such conclusions coincide with the literature data.

A large decrease in oocytes expression after IVM was observed in the gene CCND2gene and it belongs to " regulation of cell proliferation" ontological group. CCND2 is a gene coding for the cyclin D2 protein, belongs to the family of cyclin D, which is responsible for the regulation of specific cyclin-dependent kinases and the activator of cell cycle progression. CCND2 is a gene coding for the cyclin D2 protein, it belongs to the cyclin D family, which is responsible for the regulation of specific cyclin-dependent kinases and the activator of cell cycle progression. This gene affects the function of the ovaries and therefore the relationship between the expression of this gene and the differentiation of the female sex [28]. Our research has shown a significant reduction of CCND2 during IVM, these results are also confirmed by Ożegowska et al. and Robker et al. [28,29]. The obtained results and literature data may indicate that CCND2 may be a marrow for the unexpected stages of oocyte maturation.

Another gene that deserves attention is ENDRA, in our study it is the fifth gene in terms of downregulated and was represented by the "regulation of cell proliferation" ontological group. ENDRA is the gene responsible for the production of endothelin $\mathrm{A}$ receptor and the regulation of muscle cell narrowing [30]. The endothelin A receptor is found in many cells, including smooth muscle cells, theca cells, granulosa cells and luteal cells. Activation of the endothelin A receptor by ligand binding leads to vasoconstriction and the contractile forces, which are most likely required for ovulation. According to Kawamura et al. Endothelin 1 interacting with ENDRA 
may cause the breakdown of the germinal vesicles in preovulatory oocytes [31], which may explain the decreased expression of this gene after IVM in oocytes.

Belonging to the both ontology group "regulation of cell migration" and "regulation of cell proliferation "TGFBR3 - Transforming Growth Factor Beta Receptor 3, (belonging to the TGF family) is gene which plays an important role in the development of coronary arteries as well as various blood vessels in tissues. Piotrowska et al. in their research drew the conclusion that the members of the TGF superfamily influence the essential stages of oogenesis, folliculogenesis and mammalian embryogenesis [32]. The results of our experiment showed reduced expression of this IVM gene compared to those analyzed in their immature form.

In summary, we identified several genes of porcine oocyte whose expression after IVM was significantly reduced compared to the expression before IVM. The described genes play an important role in the proliferation and / or migration of cells and affect the developmental processes occurring in oocytes.

\section{Acknowledgements}

This publication and its results are an outcome of a cooperation between Poznan University of Medical Sciences (Poznań, Poland) and Polish Ministry of Science and Higher Education, with Institute of Advanced Sciences Sp. z o.o. (Poznań, Poland), as a part of the "Professional PhD" programme.

\section{Corresponding author}

Michał Nowicki, Department of Histology and Embryology, Poznan University of Medical Sciences, Święcickiego 6, 60-781, Poznan, Poland; email: mnowicki@ump.edu.pl.

\section{Conflict of interest statement}

The authors declare they have no conflict of interest.

\section{References}

1. Jamnongjit $M$, Hammes SR. Oocyte maturation: the coming of age of a germ cell. Semin Reprod Med. 2005;23:234-41; DOI:10.1055/s-2005-872451.

2. Mrazek M, Fulka J. Failure of oocyte maturation: Possible mechanisms for oocyte maturation arrest. Hum Reprod. 2003;18:2249-52; DOI:10.1093/humrep/deg434.

3. Hunt PA, Hassold TJ. Human female meiosis: what makes a good egg go bad? Trends Genet. 2008;24:86-93; DOI:10.1016/j.tig.2007.11.010.

4. Verlhac M-H, Terret M-E. Oocyte Maturation and Development. F1000Research. 2016;5:309; DOI:10.12688/f1000research.7892.1.

5. Levran D, Farhi J, Nahum H, Glezerman M, Weissman A. Maturation arrest of human oocytes as a cause of infertility: Case report. Hum Reprod. 2002;17:1604-9; DOI:10.1093/humrep/17.6.1604

6. Ming $\mathrm{T}$, Nielsen $\mathrm{H}$, Chen $\mathrm{Z}$. Maturation arrest of human oocytes at germinal vesicle stage. J Hum Reprod Sci. 2010;3:153; DOI:10.4103/0974-1208.74161.

7. Rybska M, Knap S, Jankowski M, Jeseta M, Bukowska D, Antosik P, Nowicki M, Zabel M, Kempisty B, Jaśkowski JM. Cytoplasmic and nuclear maturation of oocytes in mammals - living in the shadow of cells developmental capability. Med J Cell Biol. 2018;6:13-7; DOI:10.2478/ acb-2018-0003.

8. Gilchrist R., Ritter L., Armstrong D. Oocyte-somatic cell interactions during follicle development in mammals. Anim Reprod Sci. 2004;8283:431-46; DOI:10.1016/j.anireprosci.2004.05.017.

9. Li R, Norman RJ, Armstrong DT, Gilchrist RB. Oocyte-secreted factor(s) determine functional differences between bovine mural granulosa cells and cumulus cells. Biol Reprod. 2000;63:839-45.

10. Macaulay AD, Gilbert I, Caballero J, Barreto R, Fournier E, Tossou P, Sirard M-A, Clarke HJ, Khandjian ÉW, Richard FJ, Hyttel P, Robert C. The Gametic Synapse: RNA Transfer to the Bovine Oocyte1. Biol Reprod. 2014;91:90; DOI:10.1095/biolreprod.114.119867.
11. Antosik P, Kempisty B, Jackowska M, Piotrowska H, Bukowska D, Wozna M, Lianeri M, Brussow K-P, Jaskowski JM. Assessment of transcript and protein levels contributing to cell cycle control and gap junction connections in morphologically variable groups of porcine cumulus-oocyte complexes. Vet Med (Praha). 2010;55:512-21.

12. Chang CL, Wang H-S, Soong Y-K, Huang SY, Pai SY, Hsu SYT. Regulation of Oocyte and Cumulus Cell Interactions by Intermedin/Adrenomedullin 2. J Biol Chem. 2011;286:43193-203; DOI:10.1074/jbc.M111.297358.

13. Huang DW, Sherman BT, Tan Q Collins JR, Alvord WG, Roayaei J, Stephens R, Baseler MW, Lane HC, Lempicki RA. The DAVID Gene Functional Classification Tool: a novel biological module-centric algorithm to functionally analyze large gene lists. Genome Biol. 2007;8:R183; DOI:10.1186/gb-2007-8-9-r183.

14. Walter W, Sánchez-Cabo F, Ricote M. GOplot: An R package for visually combining expression data with functional analysis. Bioinformatics. 2015;31:2912-4; DOI:10.1093/bioinformatics/btv300.

15. von Mering C, Jensen LJ, Snel B, Hooper SD, Krupp M, Foglierini M, Jouffre N, Huynen MA, Bork P. STRING: known and predicted protein-protein associations, integrated and transferred across organisms. Nucleic Acids Res. 2004;33:D433-7; DOI:10.1093/nar/gki005.

16. Yokota Y, Mori S. Role of Id family proteins in growth control. J Cell Physiol. 2002;190:21-8; DOI:10.1002/jcp.10042.

17. Massari ME, Murre C. Helix-loop-helix proteins: regulators of transcription in eucaryotic organisms. Mol Cell Biol. 2000;20:429-40.

18. Lee SB, Frattini V, Bansal M, Castano AM, Sherman D, Hutchinson K, Bruce JN, Califano A, Liu G, Cardozo T, Iavarone A, Lasorella A. An ID2-dependent mechanism for VHL inactivation in cancer. Nature. 2016;529:172-7; DOI:10.1038/nature16475.

19. Roschger C, Cabrele C. The Id-protein family in developmental and cancer-associated pathways. Cell Commun Signal. 2017;15:7; DOI:10.1186/ s12964-016-0161-y.

20. Trau HA, Brännström M, Curry TE, Duffy DM. Prostaglandin E2 and vascular endothelial growth factor A mediate angiogenesis of human ovarian follicular endothelial cells. Hum Reprod. 2016;31:dev320; DOI:10.1093/humrep/dev320.

21. Celichowski P, Nawrocki MJ, Dyszkiewicz-Konwińska M, Jankowski M, Budna J, Bryja A, Kranc W, Borys S, Knap S, Ciesiółka S, Jeseta M, Piasecka-Stryczyńska K, Khozmi R, Bukowska D, Antosik P, Brüssow KPKP, Bruska M, Nowicki M, Zabel M, Kempisty B. "Positive Regulation of RNA Metabolic Process" Ontology Group Highly Regulated in Porcine Oocytes Matured In Vitro : A Microarray Approach. Biomed Res Int. 2018;2018:1-10; DOI:10.1155/2018/2863068.

22. Borys S, Brązert M, Jankowski M, Kocherova I, Ożegowska K, Celichowski P, Nawrocki MJ, Kranc W, Bryja A, Kulus M, Jeseta M, Pieńkowski W, Bręborowicz A, Bukowska D, Antosik P, Pawelczyk L, Skowroński MT, BRÜSSOW KP, Bruska M, Zabel M, Nowicki M, Kempisty $\mathrm{B}$. Enzyme linked receptor protein signaling pathway is one of the ontology groups that are highly up-regulated in porcine oocytes before in vitro maturation. J Biol Regul Homeost Agents. 2018;32:21-35.

23. Chermuła B, Brązert M, Jeseta M, Ożegowska K, Sujka-Kordowska $P$, Konwerska A, Bryja A, Kranc W, Jankowski M, Nawrocki M, Kocherova I, Celichowski P, Borowiec B, Popis M, Budna-Tukan J, Antosik P, Bukowska D, Brussow K, Pawelczyk L, Bruska M, Zabel M, Nowicki M, Kempisty B. The Unique Mechanisms of Cellular Proliferation, Migration and Apoptosis are Regulated through Oocyte Maturational Development-A Complete Transcriptomic and Histochemical Study. Int J Mol Sci. 2018;20:84; DOI:10.3390/ijms20010084.

24. Chaves RN, Duarte ABG, Rodrigues GQ Celestino JJH, Silva GM, Lopes CAP, Almeida AP, Donato MAM, Peixoto CA, Moura AAA, Lobo CH, Locatelli Y, Mermillod P, Campello CC, Figueiredo JR. The Effects of Insulin and Follicle-Simulating Hormone (FSH) During In Vitro Development of Ovarian Goat Preantral Follicles and the Relative mRNA Expression for Insulin and FSH Receptors and Cytochrome P450 Aromatase in Cultured Follicles1. Biol Reprod. 2012;87:69; DOI:10.1095/ biolreprod.112.099010.

25. Jones RL, Pepling ME. KIT signaling regulates primordial follicle formation in the neonatal mouse ovary. Dev Biol. 2013;382:186-97; DOI:10.1016/j.ydbio.2013.06.030.

26. Cadoret $V$, Frapsauce $C$, Jarrier $P$, Maillard $V$, Bonnet $A$, Locatelli $Y$, Royère D, Monniaux D, Guérif F, Monget P. Molecular evidence that follicle development is accelerated in vitro compared to in vivo. Reproduction. 2017;153:493-508; DOI:10.1530/REP-16-0627.

27. Chermuła B, Brązert M, Jeseta M, Ożegowska K, Kocherova I, Jankowski M, Celichowski P, Sujka-Kordowska P, Konwerska A, Piotrowska-Kempisty $\mathrm{H}$ Budna-Tukan J, Antosik P, Bukowska D, Machatkova M, Brussow KP, Skowroński MT, Pawelczyk L, Bruska M, Nowicki M, Kempisty B. Transcriptomic Pattern of Genes Regulating Protein Response and Status of Mitochondri al Activity Are Related to Oocyte Maturational Competence-A Transcriptomic Study. Int J Mol Sci. 2019;20:2238; DOI:10.3390/ijms20092238. 
28. Ożegowska K, Dyszkiewicz-Konwińska M, Celichowski P, Nawrocki MJ, Bryja A, Jankowski M, Kranc W, Brązert M, Knap S, Jeseta M, Skowroński MT, Bukowska D, Antosik P, Brüssow KP, Breborowicz A, Bruska M, Nowicki M, Pawelczyk L, Zabel M, Kempisty B. Expression pattern of new genes regulating female sex differentiation and in vitro maturational status of oocytes in pigs. Theriogenology. 2018;121:122-33; DOI:10.1016/j.theriogenology.2018.08.019.

29. Robker RL, Richards JS. Hormone-induced proliferation and differentiation of granulosa cells: a coordinated balance of the cell cycle regulators cyclin D2 and p27Kip1. Mol Endocrinol. 1998;12:924-40; DOI:10.1210/ mend.12.7.0138.

30. Bridges PJ, Cho J, Ko C. Endothelins in regulating ovarian and oviductal function. Front Biosci (Schol Ed). 2011;3:145-55

31. Kawamura K, Ye Y, Liang CG, Kawamura N, Gelpke MS, Rauch R, Tanaka T, Hsueh AJW. Paracrine regulation of the resumption of oocyte meiosis by endothelin-1. Dev Biol. 2009;327:62-70; DOI:10.1016/j. ydbio.2008.11.033.

32. Piotrowska H, Kempisty B, Sosinska P, Ciesiolka S, Bukowska D, Antosik P, Rybska M, Brussow KP, Nowicki M, Zabel M. The role of TGF superfamily gene expression in the regulation of folliculogenesis and oogenesis in mammals: a review. Vet Med (Praha). 2013;58:505-15. 